\title{
Aplicación de la ley de Lotka en los trabajos de ascenso del personal académico de la universidad nacional abierta. En el período 2000-2004
}

doi: $10.33264 /$ rpa.201901-03

\author{
MSc. Fernando Antonio Salas Granado \\ Escuela de Bibliotecología y Archivología \\ Facultad de Humanidades y Educación \\ Universidad Central de Venezuela
}

\section{Resumen}

Las bibliotecas han evolucionado desde la cuantificación de los datos hacia el interés por interpretarlos y utilizarlos como instrumentos de evaluación, los métodos bibliométricos son una herramienta eficaz que facilita el suministro de indicadores de producción. La aplicación de indicadores métricos, va más allá de los datos fijos de la estadística, esta permite una evaluación dinámica del objeto en estudio, la medición del impacto, la eficacia de las actividades investigativas, con lo cual habrá accesibilidad, objetividad y precisión de las citas para la elaboración y sustentación de las ideas expuestas por los investigadores.

Palabras clave: bibliometría, ley de Lotka, análisis de citas.

\begin{abstract}
Libraries have evolved from the quantification of data to the interest to interpret them and use them as assessment instruments, bibliometric methods are an effective tool that facilitates the supply of production indicators. The application of metric indicators, goes beyond the fixed data of the statistics, this allows a dynamic evaluation of the object under study, the measurement of the impact, the effectiveness of the research activities, with which there will be accessibility, objectivity and precision of the appointments for the elaboration and sustentation of the ideas exposed by the investigators.
\end{abstract}

Keywords: Bibliometrics, law of Lotka, analysis of appointments. 


\section{Introducción}

En la cultura Borgiana del Aleph, donde reivindican la existencia de una esfera tornasol, capaz de trasladar al usuario a otras latitudes, permitirle una visión de $360^{\circ}$ de conocimientos, esperando a ser consultados, procesados y citados. Donde miles de autores, que han procurado plasmar sus ideas, reales o ficticias para enriquecer el alma de cada individuo, para algunos, la simple sensación de deslizar los dedos al pasar cada hoja de un libro, está siendo agradecido por ellos. Bien sea documentos, libros o audiovisuales, que formen parte de la colección de una Biblioteca o Archivo, están ahí, a la espera de serles útiles a quienes afanosamente andan buscando datos referenciales que les permita concretar en buenos términos sus trabajos de investigación.

Las citas, son el factor de impacto que da a conocer que se está investigando y produciendo actualmente, determina las fortalezas y debilidades de la estructura investigativa de las Universidades, cabe destacar, que la evolución de las bibliotecas, es responsabilidad de los académicos quienes deben divulgar cuáles son sus necesidades informativas, sugerir temas idóneos para su formación y posterior aporte de conocimientos a través del aprendizaje e intercambio de ideas.

Cada individuo es una capsula de conocimiento tácito, en el cual debe existir un acompañamiento, promoción, apoyo financiero, técnico y logístico, para que se pueda generar un conocimiento explicito entre los pares y su entorno académico. El grado de actualización de una información se mide como el tiempo que ha pasado desde su disponibilidad hasta su captación, procesamiento, distribución y productividad, todo esto permite gerenciar, gestionar y tener las bases fundamentales para la tomas de decisiones a mediano plazo. "Conocer como están siendo utilizados los documentos que componen la colección”. (Sanz, 1994), es totalmente cierto que la información reposa sólo en los trabajos de ascenso desde un punto individualista del conocimiento, difícil de divulgar a los demás pares, la ambigüedad o la imprecisión de los datos disponibles, crea incertidumbre en lo referente a la producción investigativa. "Cuando un trabajo no es visible es imposible considerarlo y, por tanto, evaluarlo" (Cañedo, 1999). La disponibilidad del contenido de un documento influye necesariamente en la evaluación directa que fortalezca la producción sobre las necesidades que manifiestan los usuarios, conocer como está compuesta la colección y las ideas científicas mediante el estudio de las citas utilizadas, por un determinado autor.

La evaluación de las colecciones de una biblioteca en base a los estudios bibliométricos, permite la posibilidad de enunciar el valor agregado y la calidad de las 
publicaciones para los usuarios, estos procesos se deben llevar a cabo para comprobar hasta qué punto la colección es pertinente a las necesidades de los usuarios, el uso de los datos implica la selección y el procesamiento de la información que da como resultado un nuevo conocimiento, el cual debe ser innovador, generar nuevos resultados, dar a conocer que se está investigando, en que ámbito, las herramientas utilizadas, el impacto o factibilidad de la información.

\section{Objetivo General}

Aplicar la Ley de Lotka en los Trabajos de Ascenso del Personal Académico de la Universidad Nacional Abierta. En el período 2000-2004.

\section{Metodología}

La investigación es de tipo descriptiva-evaluativa, es un estudio delimitado y concreto, usando la recolección de los datos sobre la base de la literatura utilizada por los Académicos de la Universidad Nacional Abierta, aplicando la Ley de Lotka, como una herramienta métrica para medir la productividad científica en los Trabajos de Ascenso, para el análisis de las citas con base en la medición numérica, con la obtención de los resultados se determina que están consultando, su impacto en la comunidad Universitaria, permitiendo gestionar y planificar la obtención de recursos bibliográficos para el rendimiento de la actividad de enseñanza-aprendizaje.

\section{Cuerpo del texto}

La introducción de las matemáticas a las disciplinas sociales tiene sus antecedentes en la doctrina de Augusto Comte, filósofo y matemático francés (1798-1857), denominada positivismo, argumentando: "consiste en no admitir como válidos científicamente otros conocimientos, sino los que proceden de la experiencia, rechazando, por tanto, toda noción a priori y todo concepto universal y absoluto". Lo cual tuvo un gran impacto en los siglos XIX y XX, iniciando la matematización del conocimiento; la mayor parte de las observaciones que cimentaron el nacimiento de la Bibliometría desde fines del siglo pasado y especialmente durante la primera mitad de este proceden de investigadores ajenos al mundo bibliotecario.

El origen de los estudios métricos está enmarcado por la aparición de la Ciencia de la Documentación, cuyo propósito esencial fue a finales del siglo XIX, el control de la gran cantidad de documentos existentes en las diferentes áreas del saber, producto de un desarrollo acelerado de la ciencia y la técnica. 
En 1895, se celebró en Bruselas la Conferencia Bibliográfica Internacional, con el objetivo de estudiar, las fuentes bibliográficas. En esta conferencia Henri la Fontaine y Paul Otlet, ambos abogados, crearon ese mismo año, el Instituto Internacional de Bibliografía, luego fue denominado Oficina Internacional de Bibliografía y actualmente se conoce como Federación Internacional para la Información y la Documentación (FID).

"La idea del valor agregado se incorpora a los procesos informacionales y se concibe como un proceso de innovación” (Moros, 2003). Por lo tanto, el grado de actualización de una información se mide como el tiempo que ha pasado desde su disponibilidad hasta su captación, procesamiento, distribución y productividad, todo esto permite gerenciar, gestionar y tener las bases fundamentales para la tomas de decisiones a mediano plazo, que permite trabajar en un ambiente donde la inteligencia competitiva tecnológica, representa una práctica de enseñanzaaprendizaje, coordinada, selectiva, de análisis, con el objetivo de obtener respuestas y generar conocimientos en las áreas investigativas en las que se está trabajando.

Para (Astudillo, López, \& Rodríguez, 2004), “la aplicación de la Bibliometría, en los trabajos de investigación es muy utilizada para medir la cantidad y el impacto de las citaciones". Siendo un modelo matemático para el desarrollo de la búsqueda, interpretación, evaluación cualitativa y cuantitativa de la información, los estudios métricos son utilizados para determinar los diferentes patrones de obsolescencia de los documentos, dispersión, productividad, visualización y ranking de los trabajos de investigación, revistas, tesis, libros y todo lo que sea citado por los investigadores.

Alfred James Lotka (1880-1949), considera: “el número de trabajos publicados sobre un tema, la evolución cronológica sobre una disciplina o subdisciplina, así como también, las lagunas (falta de información) o riadas (superabundancia temática)". Lotka, químico y matemático, fundamento el estudio de la productividad de los autores y formuló la Ley Cuadrática Inversa de la Productividad de los Autores, esta ley establece que partiendo del número de autores con un solo trabajo en un tema determinado, es posible predecir el número de autores con " $n$ " trabajos con la siguiente formula:

\section{$A_{n}=A_{1} / n^{2}$}

Donde An, es el número de autores con " $n$ " citas; “A1”, es el número de autores con una cita, y “nz”, es el número de trabajos al cuadrado. 
Para un estudio métrico en cualquier área del conocimiento es necesario definir los indicadores, la creación de una base de datos en excel, siendo una herramienta necesaria para el vaciado, ordenamiento y recuperación de los datos a estudiar, delimitando las citas obtenidas de una revisión manual, en once campos: autor principal, título de la tesis, autores citados, títulos citados, editorial, año de publicación, ciudad o país, idioma, tipo de material (gacetas, revistas, digital, impreso, audiovisual, y total de citas.

El crecimiento de los trabajos de ascenso esta formalmente establecido en el Capítulo II, Artículo 24, del Reglamento Interno de la Universidad Nacional Abierta, de conformidad con lo establecido en el artículo 29 de la Ley Orgánica de Educación y el 91 de la Ley de Universidades (Universidad Nacional Abierta, 1990).

El grado de actualización de una información se mide como el tiempo que ha pasado desde su disponibilidad hasta su captación, procesamiento, distribución, productividad, lo cual permite gestionar, promover, afianzar y tener las bases fundamentales para la toma de decisiones a mediano plazo.

Los 95 Trabajos de Ascenso, demuestra que el área de mayor relevancia temática ha sido la de Educación, con 48 trabajos publicados, Administración con 29 y por último Ingeniería con 18 trabajos.

Gráfico n¹: Total de trabajos de ascenso presentados durante los años 2000-2004

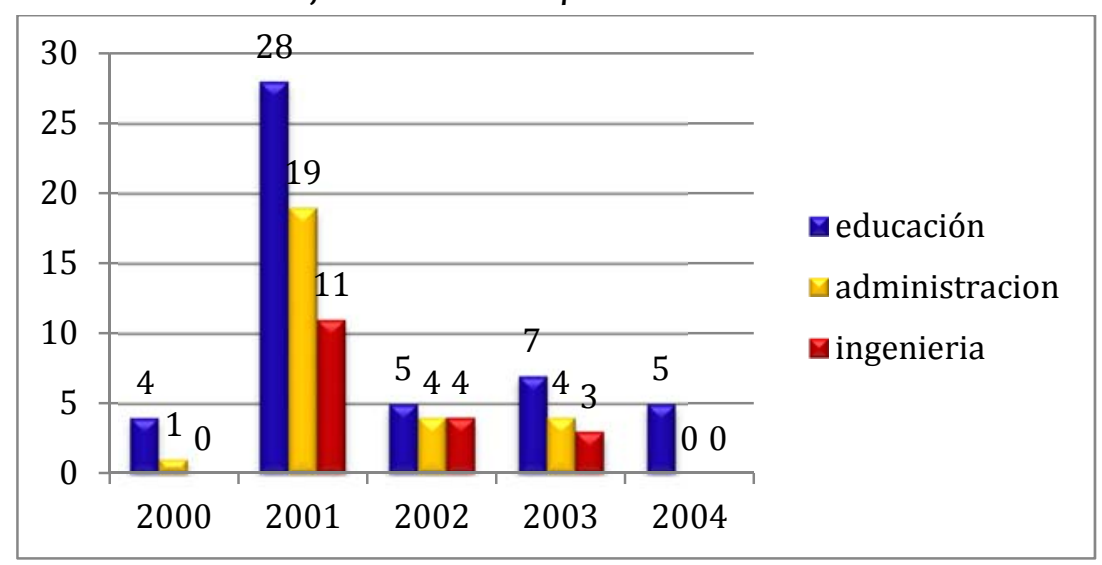

Elaboración propia

Con la aplicación de la Ley de Lotka, de un total de 4022 autores citados, sólo 3800 de los autores representan un valor significativo de producción científica durante los cinco años analizados con un 94,48\%, quedando 222 autores por debajo del nivel de producción con $5,52 \%$. 
El gráfico ${ }^{\circ} 2$, representa la totalidad general de las fuentes bibliográficas consultadas por el personal académico de la UNA, durante un período de cinco años, el 2001 fue relevante, por la presentación de cincuenta y ocho (58) trabajos de ascenso presentados, donde se citaron dos mil seis cientos veinte y seis (2626) autores.

Gráfico n²: Total de fuentes bibliográficas citadas durante los años 2000-2004

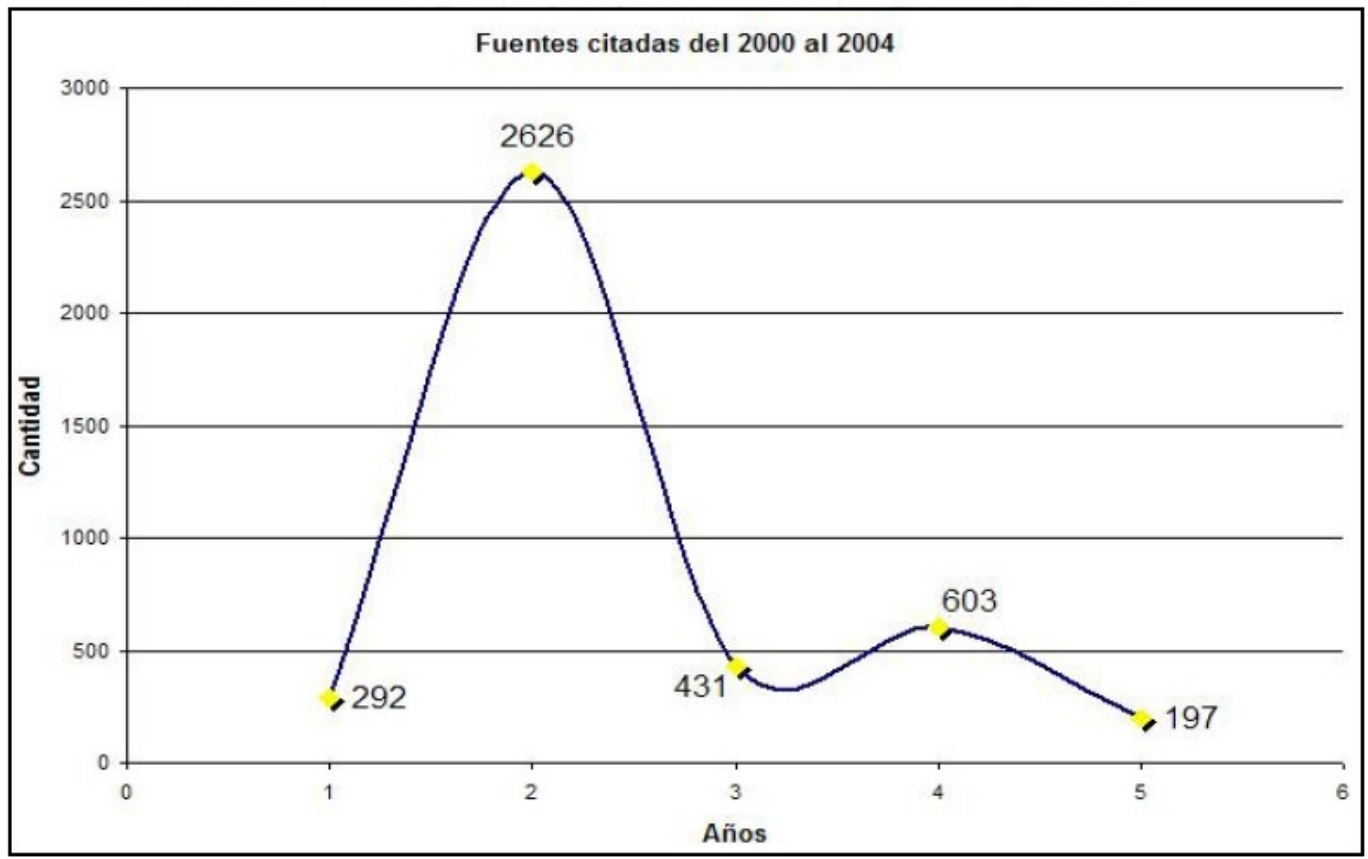

Elaboración propia

El aumento progresivo de los Trabajos de Ascenso, durante el año 2001, es producto de la oportunidad de los aspirantes en calidad de contratados a formar parte de la Universidad Nacional Abierta, la entrega de un trabajo de investigación por parte de los docentes era un requisito obligatorio.

Con la aprobación de la Comisión Calificadora de la UNA, solicitada por el Vicerrector Académico Manuel Castro Pereira, se aprobó y publicó en la Resolución N C.D.-1846, del acta $\mathrm{N}^{\circ}$ O-27, el día 1 de agosto de 2002, la necesidad de agilizar el proceso de estudio de las solicitudes de Ascenso, con lo cual no es necesario presentar un trabajo de investigación, sólo necesitan presentar una prueba escrita y una defensa oral de un tema que es asignado por el Vicerrectorado Académico, estos son opcionales en los casos de que se amerite la presentación del mismo.

La brecha informacional que persiste entre los Docentes y las Bibliotecas, transciende en el tiempo, los Bibliotecarios somos considerados herederos de los escribanos, sólo aceptamos fielmente ordenes, la mayoría de las teorías, leyes, estatutos, 
nomenclaturas, ordenamiento, clasificación y cálculo matemático a formado parte de la inquietud de otros investigadores. Cabe destacar, que a los responsables de promover el conocimiento, esa triada que debe existir entre Docentes-UniversidadBibliotecas, se les debe preguntar: ¿Este trabajo es necesario para aportar conocimiento? ¿Está contribuyendo al rendimiento académico? ¿La colección de las bibliotecas, les aporta el apoyo necesario para el rendimiento académico investigativo? ¿Qué podemos aprender de ustedes? ¿Qué nos pueden decir de su labor investigativa y como realizarla? ¿Qué herramientas necesitan? ¿Qué información necesitan? ¿Su aporte intelectual, tiene valor agregado para la comunidad universitaria?

El conocimiento, exige un aprendizaje continuo porque está evolucionando constantemente, es pertinente acotar que durante el siglo XIX, Gregor Mendel (18221884), genetista y científico adelantado para su época, patento las Leyes de la Genética; donde el cruce de los cromosomas de dos parejas conllevan a la conformación de un ser vivo, independientemente si es animal o humano, lo primordial es que la nueva generación tendrá las características de ambos portadores. Muchas veces, escucharemos, ¡El conocimiento es Poder, y no lo compartiré con nadie, lo que he aprendido vale mucho! Muy bien por el que piensa así, simplemente se quedara varado en la orilla, enredado en su maraña de pensamiento inquisidor.

Las Bibliotecas, son un conglomerado de saberes, cada libro es un gen egoísta, los datos, información, formulas, guías, mapas, rastros, trozos de palabras y oraciones, que se esconden mucho más que la isla del tesoro, cada uno de nosotros nos parecemos a Robinson Crusoe, otras tantas, somos descendientes de Marco Polo, buscamos una aguja en el pajar, para seguir nuestro trayecto de investigación en otras latitudes, países, idiomas, y diferentes panorámicas de saberes.

Somos invitados a un templo de infinidades aportes prácticos, filosóficos, altruistas, emblemáticos, lleno de fabulas, verdades e hipótesis en espera de que otros continúen con el legado del autor o lo mejoren. En su momento ellos estaban adelantados en su tiempo, tal vez fueron emprendedores, porque deseaban demostrar que sus propuestas no eran locuras y tampoco ameritaban que los inquisidores los quemaran para ocultar sus ideas.

Fueron pioneros para un sector ignorante, que engullía todo lo que ellos producían y tomaban posesión de ello, creando cofradías, movimientos protestantes, porque solo la verdad absoluta les pertenecía a ellos y se debía repetir una y otra vez hasta que las personas fuesen condicionadas y hayan creído que era real. 
Todo es cuestión de adaptabilidad, compartir exponencialmente, multiplicar la información recibida, no importa las fronteras, no necesitamos visa para poder conversar, sugerirle, solicitarle amablemente información pertinente a otros compañeros bibliotecarios o archivólogos, para que sea procesada y divulgada a otros.

Algunas aves no deben estar encerradas, tal vez en este arduo camino, hemos experimentado ser como Andy Dufresne, silenciosos, precavidos, avocados en trabajar, cumplir un horario, jóvenes que deseamos comernos el mundo. Un mundo, que nos abre sus puertas desde el momento que nos graduamos, un título que en realidad nos enseñan a ser empleados y no que seamos emprendedores; esta segunda opción, no la aprendes en un salón de clase, sentado cualquier cantidad de horas en un pupitre y horas de trasnocho tratando de memorizar, autores, frases, años, paginas, bases de datos y con el sueño de aspirar una mejor calidad de vida.

En ocasiones, los árboles no nos dejan ver el bosque, la exuberante proliferación de artefactos tecnológicos que no dejan de aparecer, de crecer y que no nos dejan ver realmente el fenómeno que estamos viviendo. Casi nos identificamos como el personaje del librero de Kabul, Sultan Khan, ha tenido que hacer auténticos milagros para seguir adelante con su actividad.

Algunos dirán que los bibliotecarios, somos servidores de información, referencistas o que estamos institucionalizados esperando nuestra jubilación y luego de pasar ese capítulo ¿Qué somos? ¿Qué haremos? ¿Conformaremos una empresa? ¿Viajaremos?, aún no está escrito, pero algo es muy cierto, si tienes el valor de dejar atrás todo lo que te protege y te consuela, lo cual puede ser cualquier cosa como tu casa o viejos rencores, y embarcarte en un viaje en búsqueda de conocimientos, en las letras mágicas contenidas en cada libro, audiovisual, pinceladas, esculturas; es un arte, es conocimiento. 


\section{Referencias}

Astudillo, G., López, R., \& Rodríguez, M. (2004). Análisis cienciométrico de la producción de conocimiento científico a partir de los trabajos de ascenso, postgrado e investigación (1970-2002) de la Facultad de Arquitectura y Urbanismo. Caracas: Universidad Central de Venezuela.

Cañedo, R. (1999). Los análisis de citas en la evaluación de los trabajos científicos y las publicaciones seriadas. ACIMED, 1(9), 30.

López, P. (1996). Introducción a la bibliometría. Valencia, España: Promolibros.

Moros, Á. (2003). Evolución del concepto de conocimiento desde la pirámide informacional: propuesta de servicio de gestión del conocimiento científico para instituciones de educación supeior. Madrid: Universidad Carlos III.

Nonaka, I., \& Takeuchi, H. (1995). The knowledge-creating company: how Japanese companies create the dynamics of innovation. Recuperado el 05 de 07 de 2018, de http://www.sopper.dk/speciale/arkiv/ni95.pdf

Sanz, E. (1994). Manual de estudio de usuarios. Madrid: Fundación Germán Sánchez Ruipérez.

Universidad Nacional Abierta. (19 de Noviembre de 1990). Universidad Nacional Abierta. Obtenido de http://biblo.una.edu.ve/biblodig/bdpubuna/bdregnor/ bdtextos/19901119-cs066.pdf

\begin{tabular}{|l|}
\hline Fernando Antonio Salas Granado \\
Licenciado en Bibliotecología - Universidad Central de Venezuela - UCV \\
MSc. Gerencia de Recursos Humanos - Universidad Nacional Experimental Rafael María \\
Baralt - UNERMB \\
Experto en E-Learning - Fundación para la Actualización Tecnológica de Latinoamérica - \\
FATLA \\
Coordinador de Biblioteca - Universidad Nacional Abierta - Centro Local Falcón, Venezuela
\end{tabular}

